\title{
Odontalgia não odontogênica: levantamento e revisão da literatura atual
}

\author{
Non-odontogenic toothache: survey and revision of the current literature \\ Odontalgia no odontogénica: levantamiento y \\ revisión de la literatura actual \\ José Alcides Almeida de ARRUDA ${ }^{1}$ \\ Leni Verônica Oliveira SILVA ${ }^{1}$ \\ Amália MORENO ${ }^{1}$ \\ Ricardo Alves MESQUITA \\ Gerhilde CALLOU ${ }^{2}$
}

${ }^{1}$ Departamento de Clínica, Patologia e Cirurgia Odontológicas, Faculdade de Odontologia, UFMG

Universidade Federal de Minas Gerais, 31270-010 Belo Horizonte - MG, Brasil

${ }^{2}$ Departamento de Patologia Bucal, Faculdade de Odontologia, UPE, Universidade de Pernambuco, 54753-020 Camaragibe - PE, Brasil

\begin{abstract}
Resumo
Introdução: Apesar da maior compreensão acerca da dor orofacial nos últimos anos, o diagnóstico preciso da dor ainda é um desafio na odontologia contemporânea. Muitos distúrbios na região de cabeça e pescoço são reconhecidos para se referir à dor de estruturas dentárias ou que simulam a odontalgia. Objetivo: O objetivo desse estudo foi realizar uma revisão de literatura atual de séries e/ou relatos de casos de pacientes portadores de odontalgia de natureza não odontogênica a fim de contribuir na elucidação dos diagnósticos diferenciais e discutir os principais achados clínicos. Material e Método: A revisão da literatura foi elaborada em Agosto de 2017 com base em artigos publicados na língua inglesa, do banco de dados Medline (PubMed). Foram utilizadas pesquisas do período compreendido entre 1968 e 2016 para determinar o mapeamento dos casos dos pacientes. Os dados foram arquivados em ano da publicação, idade, gênero, número de casos, localização anatômica e diagnóstico diferencial. Resultados: Vinte e quatro artigos foram analisados. Diferentes diagnósticos e condutas foram tomadas nas odontalgias não odontogênicas. Conclusão: É pertinente concluir que o diagnóstico diferencial entre a odontalgia e as doenças de natureza não odontogênica é imprescindível a fim de promover aos pacientes tratamentos conservadores, quando possíveis e melhores prognósticos evitando sobremaneira a mutilação dos dentes, tratamentos endodônticos desnecessários e terapêuticas tardias nos casos de neoplasias malignas.

Descritores: Odontalgia; Dor facial; Diagnóstico.
\end{abstract}

\begin{abstract}
Introduction: Despite the increased understanding about orofacial pain in recent years, the accurate diagnosis of pain is still a challenge in contemporary dentistry. Many disturbances in head and neck region are recognized to refer to pain of dental structures or that simulate the toothache. Objective: The aim of this study was to conduct a review of current literature series and/or case reports of patients with toothache of non-odontogenic in order to contribute to the elucidation of the differential diagnoses and discuss the main clinical features. Material and Method: The literature review was realized in November 2016 based on articles published in the English language of database Medline (PubMed). Research used the period between 1968 and 2016 to determine the mapping of cases of patients. The data were archived in the publication year, age, gender, number of cases, anatomical location and differential diagnosis. Results: Twenty-four articles were analyzed. Differential diagnosis and conduct were taken in toothache of non-odontogenic. Conclusion: It is pertinent to conclude that the differential diagnosis between the toothache and non-odontogenic diseases is essential in order to promote conservative treatments to patients, when possible and better prognoses avoiding the teeth mutilation, endodontic treatments and unnecessary late therapeutic in cases of malignant neoplasms. Descriptors: Toothache; Facial Pain; Diagnosis
\end{abstract}

\section{Resumen}

Introducción: A pesar que en los últimos años, aspectos acerca del dolor orofacial han sido mejor entendidos, el diagnóstico exacto del dolor sigue siendo un reto en la odontología contemporánea. Muchos trastornos en la región de la cabeza y cuello son atribuidos al dolor de estructuras dentales y que son confundidos con la odontalgia. Objetivo: El objetivo del presente estudio fue realizar una revisión de literatura actual de artículos y de relatos de casos de pacientes que sufren de odontalgia de naturaleza no odontogénica, con el fin de contribuir al mejor entendimento de diagnósticos diferenciales y discutir los principales hallazgos clínicos. Material y Método: La revisión de literatura fue elaborada en agosto del 2017 con base en artículos publicados en el idioma inglés del banco de datos Medline (PubMed). Fueron utilizadas investigaciones del período comprendido entre 1968 al 2016 para determinar el mapeo de los casos clínicos. Los datos fueron archivados según el año de publicación, edad, género, número de casos, ubicación anatómica y diagnóstico diferencial. Resultados: Veinticuatro artículos fueron analizados. Diferentes diagnósticos y conductas fueron determinadas en los casos de odontalgias no odontogénicas. Conclusión: Es pertinente concluir que el diagnóstico diferencial entre la odontalgia y las enfermedades de naturaleza no odontogénica es imprescindible con el fin de promover tratamientos conservadores, cuando son posibles y determinar mejores pronósticos a los pacientes, de esa forma evitar mutilaciones excesivas de dientes, tratamientos endodónticos innecesários y terapias tardias en casos de neoplasias malignas.

Descriptores: Odontalgia; Dolor Facial; Diagnóstico

\section{INTRODUÇÃO}

A dor pode ser a pior das experiências humanas. Às vezes é apenas irritante, mas em outras é penosa a ponto do paciente perder a consciência quando seu sistema não pode suportar a duração da experiência. De todos os sintomas que o cirurgião-dentista enfrenta, a dor é a mais pungente e livrar o paciente da dor é talvez o aspecto mais gratificante da prática ${ }^{1-3}$. Normalmente, a experiência da dor é iniciada em uma base fisiológica via sistema periférico. Na região dos maxilares, as fibras nociceptoras são componentes do nervo trigêmeo. $\mathrm{O}$ corpo celular de todas essas fibras se localiza no Gânglio de Gasser e os axônios aferentes que nutrem estas células saem do gânglio e estendem-se em direção ao sistema nervoso central através do tronco do trigêmeo que penetra na ponte. Essas fibras, então, partem da ponte inferiormente para a parte mais alta da região cervical da medula espinhal. Como regra, o paciente está apto a localizar a região específica da dor, onde o tecido abriga o processo patológico que produziu a sensação de dor. Quando o profissional não está bem atento, a dor aguda 
e intensa nem sempre pode ser prontamente localizada. A base neuroanatômica para a capacidade de localizar especificamente a dor intensa é ainda mal compreendida. Às vezes, o profissional pode encontrar sintomatologia dolorosa referida aos dentes e maxilares, porém de natureza não odontogênicas ${ }^{1-3}$.

Apesar da maior compreensão acerca da dor orofacial nos últimos anos, o diagnóstico preciso da dor ainda é um desafio na odontologia contemporânea. Muitos distúrbios na região de cabeça e pescoço são reconhecidos para se referir à dor de estruturas dentárias ou que simulam a odontalgia ${ }^{4}$. Pouca investigação tem havido sobre o problema latente da correlação entre a dor miofacial (a mais frequente dor de origem não odontogênica) que pode simular sintomas de, por exemplo, pulpites.

Com base no exposto, o objetivo desse trabalho foi realizar uma revisão de literatura com artigos publicados de séries e/ou relatos de casos de pacientes portadores de odontalgia de natureza não odontogênica a fim de contribuir na elucidação dos diagnósticos diferenciais.

\section{MATERIAL E MÉTODO}

A revisão de literatura foi realizada em Agosto de 2017 por meio de artigos da língua inglesa, do banco de dados Medline (PubMed) relacionados a séries e/ou relatos de casos em pacientes portadores de odontalgia de natureza não odontogênica. Os seguintes descritores foram utilizados para busca: dor de origem não odontogênica e dor não odontogênica. Pacientes fora da faixa etária de adultos, adultos jovens e idosos foram excluídos. Relatos de casos que não estavam relacionados a odontalgia de natureza não odontogênica, trabalhos duplicados com diferentes descritores e sem resumos foram excluídos.

\section{RESULTADOS}

Os artigos foram publicados na literatura no período compreendido entre 1968 a 2016 na língua inglesa foram analisados para determinar o mapeamento dos casos de pacientes portadores de odontalgia de natureza não odontogênica. $\mathrm{O}$ arquivo de busca Medline (Pubmed) foi utilizado com os seguintes descritores: dor de origem não odontogênica e odontalgia não odontogênica.

Os casos, contabilizados e separados por séries e/ou relatos de casos. Noventa e um trabalhos foram levantados, dos quais, 67 foram excluídos. Os 24 trabalhos restantes, selecionados de acordo com o critério de inclusão estão distribuídos na Tabela 1.

\section{DISCUSSÃO}

As queixas de dor na região orofacial são, em sua maioria, de origem odontogênica. Entretanto, os dentistas podem enfrentar situações em que os pacientes apresentam como queixa principal uma dor que supostamente é de odontogênica, mas muitas vezes após anamnese e exames adequados descobrem estar relacionadas a disfunções temporomandibulares. Mosaferi et al. ${ }^{6}$ apresentaram um relato de caso de uma paciente que se queixava de dor na região anterior da maxila. Ao exame clínico, observou-se fístula com drenagem em região vestibular. Ao exame radiográfico, levantou-se inicialmente como diagnóstico lesão periapical relacionada aos incisivos. Após investigações médicas completas, concluiu-se que se tratava de osteonecrose, como efeito colateral, da utilização do bifosfonato.

Como defendem Renton e Egbuniwe $^{7}$ o desconhecimento da dor crônica da disfunção pode
Tabela 1. Dados dos casos de odontalgia não odontogênica publicados no PubMed-Medline no período de 1968 a 2016

\begin{tabular}{|c|c|c|c|c|}
\hline $\begin{array}{l}\text { Autor } \\
\text { (Ano) }\end{array}$ & Idade/Sexo & $\begin{array}{l}\text { No de } \\
\text { casos }\end{array}$ & $\begin{array}{c}\text { Localização } \\
\text { anatômica }\end{array}$ & $\begin{array}{c}\text { Diagnóstico } \\
\text { diferencial }\end{array}$ \\
\hline $\begin{array}{l}\text { Mosaferi } \\
\text { et al } \\
(2016)\end{array}$ & $52 / F$ & 1 & $\begin{array}{c}\text { Região dos } \\
\text { incisivos em } \\
\text { maxila }\end{array}$ & $\begin{array}{l}\text { Lesão } \\
\text { periapical }\end{array}$ \\
\hline $\begin{array}{l}\text { Kwon et al } \\
(2015)\end{array}$ & $70 / F$ & 1 & $\begin{array}{l}\text { Região do } \\
\text { canino direito } \\
\text { ao ângulo } \\
\text { esquerdo da } \\
\text { mandíbula }\end{array}$ & $\begin{array}{l}\text { Lesão } \\
\text { radiolúcida }\end{array}$ \\
\hline $\begin{array}{l}\text { Macdonald } \\
\text { et } \quad \text { al } \\
(2015)\end{array}$ & $\begin{array}{c}9 ; 11 ; 17 ; 26 ; 29 \\
3 F ; 2 M\end{array}$ & 5 & $\begin{array}{c}\text { Maxila e } \\
\text { mandíbula }\end{array}$ & QOM \\
\hline $\begin{array}{l}\text { Sivolella et } \\
\text { al (2015) }\end{array}$ & $52 / \mathrm{M}$ & 1 & Mandíbula & Osteomielite \\
\hline $\begin{array}{l}\text { Sethukum } \\
\text { ar et al } \\
(2015)\end{array}$ & Jovem/M & 1 & Maxila & Cisto nasolabial \\
\hline $\begin{array}{l}\text { Webber et } \\
\text { al (2015) }\end{array}$ & - & 1 & $\begin{array}{l}\text { Região anterior } \\
\text { de maxila }\end{array}$ & $\begin{array}{l}\text { Linfoma de } \\
\text { células B não- } \\
\text { Hodgkin }\end{array}$ \\
\hline $\begin{array}{l}\text { Ahmadieh } \\
\text { et } \quad \text { al } \\
(2014)\end{array}$ & $46 / F$ & 1 & Mandíbula & $\begin{array}{l}\text { Lesão } \\
\text { mandibular }\end{array}$ \\
\hline $\begin{array}{l}\text { Vasudeva } \\
\mathrm{n} \text { et al } \\
(2014)\end{array}$ & $75 / \mathrm{M}$ & 1 & $\begin{array}{c}\text { Região de } \\
\text { primeiro molar } \\
\text { superior direito }\end{array}$ & $\begin{array}{l}\text { Origem pulpo- } \\
\text { periodontal }\end{array}$ \\
\hline $\begin{array}{l}\text { lino et al } \\
(2013)\end{array}$ & $36 / \mathrm{M}$ & 1 & $\begin{array}{l}\text { Região anterior } \\
\text { de maxila }\end{array}$ & $\begin{array}{c}\text { Lesão } \\
\text { periodontal; } \\
\text { cisto } \\
\text { periodontal } \\
\text { lateral } \\
\text { Carcinoma de } \\
\text { células } \\
\text { escamosas } \\
\text { intra-ósseo }\end{array}$ \\
\hline $\begin{array}{l}\text { Mohamme } \\
\text { d et al } \\
(2013)\end{array}$ & $20 / F$ & 1 & $\begin{array}{l}\text { Região de } \\
\text { corpo } \\
\text { mandibular } \\
\text { esquerdo }\end{array}$ & $\begin{array}{l}\text { Osteoma } \\
\text { osteóide }\end{array}$ \\
\hline $\begin{array}{l}\text { López- } \\
\text { López et al } \\
\text { (2012) }\end{array}$ & $\begin{array}{c}\text { 64-65;49-74/ } \\
3 \mathrm{~F} ; 8 \mathrm{M}\end{array}$ & 11 & Mandíbula & $\begin{array}{l}\text { Origem } \\
\text { cardíaca }\end{array}$ \\
\hline $\begin{array}{l}\text { Mello et al } \\
\text { (2011) }\end{array}$ & $\pm 31 / 25 \mathrm{~F} ; 21 \mathrm{M}$ & 46 & $\begin{array}{c}\text { Maxila e } \\
\text { mandíbula }\end{array}$ & QO \\
\hline $\begin{array}{l}\text { Prakash et } \\
\text { al (2010) }\end{array}$ & - & 4 & $\begin{array}{l}\text { Dores em } \\
\text { região dentária }\end{array}$ & $\begin{array}{l}\text { Hemicrânia } \\
\text { continua }\end{array}$ \\
\hline $\begin{array}{l}\text { Saund et } \\
\text { al (2010) }\end{array}$ & $38 / F$ & 1 & Maxila & $\begin{array}{l}\text { Linfoma não- } \\
\text { Hodgkin }\end{array}$ \\
\hline $\begin{array}{l}\text { August et } \\
\text { al (2003) }\end{array}$ & $40-77 / F$ & 4 & $\begin{array}{c}\text { Maxila e } \\
\text { mandíbula }\end{array}$ & $\begin{array}{l}\text { Carcinoma de } \\
\text { células claras } \\
\text { Carcinoma }\end{array}$ \\
\hline $\begin{array}{l}\text { Favia et al } \\
(2000)\end{array}$ & $46 / F$ & 1 & Mandíbula & $\begin{array}{l}\text { adenóide } \\
\text { cístico intra- } \\
\text { ósseo }\end{array}$ \\
\hline $\begin{array}{l}\text { Ribera } \\
(1996)\end{array}$ & $69 / M$ & 1 & $\begin{array}{l}\text { Região anterior } \\
\text { de maxila }\end{array}$ & $\begin{array}{c}\text { Osteoblastoma } \\
\text { benigno }\end{array}$ \\
\hline $\begin{array}{l}\text { Sulfaro } e \\
\text { Gobetti } \\
\text { (1995) }\end{array}$ & $56 / F$ & 1 & $\begin{array}{c}\text { Região } \\
\text { posterior de } \\
\text { maxila (lado } \\
\text { direito) }\end{array}$ & $\begin{array}{c}\text { Neuralgia } \\
\text { occipital }\end{array}$ \\
\hline $\begin{array}{l}\text { Law \& Lilly } \\
\text { (1995) }\end{array}$ & 72;59/F;M & 2 & $\begin{array}{c}\text { Maxila e } \\
\text { mandíbula }\end{array}$ & $\begin{array}{l}\text { Neuralgia } \\
\text { trigeminal }\end{array}$ \\
\hline & - & 2 & $\begin{array}{l}\text { Região dos } \\
\text { dentes }\end{array}$ & Dor dentária \\
\hline $\begin{array}{l}\text { Bavitz et al } \\
\text { (1992) }\end{array}$ & $46 / F$ & 1 & $\begin{array}{l}\text { Maxila (lado } \\
\text { direito) }\end{array}$ & $\begin{array}{l}\text { Linfoma não- } \\
\text { Hodgkin }\end{array}$ \\
\hline $\begin{array}{l}\text { Ellinger e } \\
\text { Kelly } \\
(1989)\end{array}$ & $31 / \mathrm{M}$ & 1 & $\begin{array}{c}\text { Maxila (lado } \\
\text { direito) }\end{array}$ & Linfoma antral \\
\hline
\end{tabular}

resultar em tratamento odontológico inadequado e danos maiores para o paciente. Adicionalmente, Nixdorf et al. ${ }^{8}$ alertaram para os casos de pacientes submetidos a tratamento endodôntico que persistem com dor crônica por mais de seis meses e que, após investigação mais acurada, concluíram o diagnóstico de dor de natureza não odontogênica. Este fato ocorre, e mais uma vez cabe se dar ênfase a importância de uma anamnese sistemática e criteriosa, a fim de se evitar tratamentos desnecessários e a postergação do início do tratamento correto submetendo o 
paciente a suportar longos períodos de dor.

Atenção especial deve ser dada aos pacientes com queixa de dor odontogênica cujo diagnóstico final pode estar relacionado com neoplasias de natureza maligna, tais como linfoma ${ }^{9-11}$, carcinoma adenóide cístico ${ }^{12}$, carcinoma odontogênico de células claras ${ }^{13,14}$, carcinoma de células escamosas intraósseo ${ }^{15}$ e linfoma de Burkitt ${ }^{16}$. Muitas vezes a queixa principal pode levar o profissional menos desavisado a realizar um tratamento baseando-se apenas na queixa de odontalgia em referido dente. Isto se deve também à negligência a testes como o de sensibilidade pulpar, a realização de exames com mais cautela e à imperativa necessidade de exames complementares, tais como: radiografias panorâmicas e tomografias computadorizadas de feixe cônico.

Em algumas situações quando a queixa de dor está associada a um aumento de volume, expansão óssea e mobilidade dental o profissional deve ficar atento e não descartar, por exemplo, a hipótese diagnóstica de carcinoma odontogênico de células escamosas ${ }^{13}$. Salienta-se ainda que, o linfoma de Hodgkin é uma condição incomum na cavidade oral e o diagnóstico precoce é desafiador já que os sinais e sintomas apresentados são, muitas vezes, semelhantes aos de infecções odontogênicas. Relato de caso descrito por Saund et al. ${ }^{11}$ exemplifica condição em que só após tratamento endodôntico, retratamento e cirurgia paraedondôntica, o paciente recebeu o diagnóstico final de neoplasia maligna. A demora na obtenção do diagnóstico em casos de neoplasias malignas pode, se não levar a óbito, promover danos irreversíveis ao paciente.

Casos extremos de exodontias foram relatados na literatura por Prakash et al. ${ }^{17}$ em pacientes com queixa de dor orofacial crônica. Para esses autores, a inter-relação de dor de cabeça com dor orofacial é elevada na população em geral. Ademais, os pacientes com dor facial muitas vezes procuram os serviços odontológicos e, infelizmente, só após vários tratamentos mal sucedidos são corretamente diagnosticados como portadores de hemicrânia paroxística. Os resultados sugerem que o tratamento inicial com medicamentos específicos para as cefaleias primárias deva ser instituídos antes de submeter os pacientes a procedimentos invasivos.

Da mesma forma, lesões císticas odontogênicas ou não devem ser levadas em consideração no diagnóstico diferencial de pacientes com queixa de dor dentária, uma vez que o cisto nasolabial ou o queratocisto odontogênico são referenciados em tal categoria. A dor pode estar presente e a queixa ser de odontalgia ou de crescimento expansivo rápido, ainda que extremamente raro $^{18,19}$. Para Gelpi et al. ${ }^{20}$, o cisto periodontal lateral é outra modalidade de diagnóstico diferencial para os pacientes portadores de odontalgia de natureza não odontogênica. Por isso a importância de não se desmerecer um diagnóstico diferencial possível, por mais simples que seja, quando da queixa de uma dor odontogênica.

Dentro da categoria de doenças incomuns, em algumas vezes a dor originada de lesão endo-periodontal é a principal queixa dos pacientes que procuram atendimento de urgência. Entretanto, uma avaliação cuidadosa deve ser realizada principalmente no diagnóstico diferencial destas patologias com outras extremamente raras, como por exemplo, a artrite temporal com artrite reumatóide, juntamente com doença pulmonar intersticial; a fim de se evitar tratamento inapropriado. A dor da artrite temporal é localizada na área anatômica da artéria e irradiada para baixo em direção ao ouvido e dentes. Tal ocorrência pode confundir o profissional e deformá-lo no sentido de valorizar a queixa de dor e desmerecer uma investigação mais profunda. Quando isso ocorre, considera-se como padrão ouro para o diagnóstico a biópsia da artéria temporal. ${ }^{21}$

Mesma situação ocorre com a doença de Gaucher que é uma condição sistêmica autossômica recessiva rara, sendo a mais comum das doenças de depósito lisossômico. Caracteriza-se pelo acúmulo de lipídeos em determinadas células e órgãos. Quando essa doença apresenta envolvimento em região de cabeça e pescoço, uma análise cuidadosa dos sinais e sintomas e históricos médicos deve ser realizada. A biópsia pode ser necessária em alguns casos, para o diagnóstico definitivo das lesões dolorosas dos maxilares e a fim de descartar outras condições odontogênicas ou não, no diagnóstico diferencial. ${ }^{22}$

Adicionalmente, no grupo de doenças inusuais, Mohammed et al. ${ }^{23}$ apresentou um caso de osteoma osteóide, que é um tumor ósseo benigno, cuja dor inexplicavelmente foi aliviada pela ingestão de analgésicos. O dilema diagnóstico no caso em questão foi agravado por sua associação incomum com os dentes. Em tais situações, os autores defenderam que o uso da tomografia computadorizada é aconselhado a fim de distinguir a odontalgia de outros tumores ósseos. Outro exemplo, relatado por Ribera ${ }^{24}$ em 1996, se refere a um paciente portador de um osteoblastoma na região de maxila anterior, com história de dor crônica orofacial por seis anos, submetido, inclusive, a tratamento endodôntico sem resolução do caso. Cirurgia exploratória revelou tratar-se também de outra lesão óssea. Esses casos referidos enfatizam a relevância do conhecimento das doenças quer sejam raras ou não no estabelecimento de diagnósticos diferenciais para os pacientes com odontalgia de natureza não odontogênica.

De acordo com López-López et al. ${ }^{25}$, os tipos mais comuns de dor orofacial se originam nas estruturas dentárias ou periodontal, bem como, nas músculo-esqueléticas. No entanto, o paciente pode apresentar odontalgia, ainda que a fonte esteja situada em outra parte do corpo. Uma possível origem de dor heterotópica é a de natureza cardíaca, que é considerada atípica devido à sua localização. Por isso, o diagnóstico diferencial de dor de origem dentária deve ser considerado juntamente com a não-odontogênica (músculo, psicogênica, neuronal, cardíaco, sinusite e neurovascular), a fim de evitar erros de diagnóstico na prática odontológica, bem como tratamentos desnecessários. Estes autores alertam que a dor de dente é descrita como pulsátil e a cardíaca como de opressão e com sensação de queimação. Adicionalmente, relatam que devem ser notificados que a intensidade da dor foi mais elevada em pacientes com odontalgia que aqueles com origem cardíaca. Quando a origem da dor cardíaca pode ser correlacionada com a orofacial o dentista tem a obrigação de encaminhar o paciente para um cardiologista com uma descrição detalhada dos dados obtidos na anamnese.

Para Sulfaro e Gobetti ${ }^{26}$ a neuralgia occipital pode ser enquadrada no grupo do diagnóstico diferencial para os pacientes portadores de odontalgia. Os autores publicaram um relato de caso na qual a paciente queixou-se de odontalgia no quadrante posterior direito da maxila e que após acompanhamento neurológico foi diagnosticado com essa raríssima patologia. Outra modalidade de neuralgia ${ }^{27}$, a trigeminal, foi identificada em dois pacientes com odontalgia, tratados endodonticamente. $\mathrm{E}$, diante da ineficácia do tratamento, devido ao erro de diagnóstico, um 
regime terapêutico com carbamazepina foi que promoveu o alívio da dor ao paciente. Este caso demonstra a importância de se ter um conhecimento profundo de ambas as causas odontogênicas e não odontogênicas da dor orofacial, bem como do cuidadoso diagnóstico antes de se iniciar qualquer tratamento. Finalmente para $\mathrm{Heir}^{28}$ as comunidades médicas e leigos tornaram-se mais conscientes do papel da odontologia no diagnóstico e manejo dos transtornos da dor orofacial.

\section{CONCLUSÃO}

A conscientização e o conhecimento do grupo de doenças que se enquadram no diagnóstico diferencial da odontalgia de natureza não odontogênica é imprescindível na tentativa de implementar tratamentos odontológicos mais conservadores, previsíveis e com melhores prognósticos. Vale ressaltar também a importância, durante a anamnese, de questionar os pacientes a cerca de quais medicamentos estão sendo ou foram administrados na sua história médica, em se tratando de doenças crônicas. Tais iniciativas evitarão diversos procedimentos desnecessários como, tratamentos endodônticos, cirurgias parendodônticas, exodontias, efeitos adversos causados por intoxicação ou interações medicamentosas, e ainda, o atraso no tratamento de pacientes portadores de dores crônicas, quer sejam de natureza maligna ou não.

\section{REFERÊNCIAS}

1. Hargreaves KM, Berman LH. Cohen's Pathways of the Pulp Expert Consult,11th ed. Mosby, Elsevier; 2015.

2. Balasubramaniam R, Turner LN, Fischer D, Klasser GD, Okeson JP. Non-odontogenic toothache revisited. OJST. 2011; 1(3):92-102.

3. Okeson JP. Bell's oral and facial pain. 7th ed. Hanover Park, Quintessence books; 2014.

4. Kim ST. Myofascial pain and toothaches. Aust Endod J. 2005; 31(3):106-10.

5. Stegenga B. [Complaints of pain in the orofacial area of non-odontogenic origin] Ned Tijdschr Tandheelkd. 2016; 123(11):519-26.

6. Mosaferi H, Fazlyab M, Sharifi S, Rahimian S. Bisphosphonate-induced osteonecrosis of the maxilla resembling a persistent endodontic lesion. Iran Endod J. 2016; 11(1):67-70.

7. Renton T, Egbuniwe O. Pain Part 5b: Non-odontogenic dysfunctional pain. Dent Update. 2015; 42(9):856-65.

8. Nixdorf DR, Law AS, John MT, Sobieh RM, Kohli R, Nguyen RH et al. Differential diagnoses for persistent pain following root canal treatment: a study in the National Dental PBRN. J Endod. 2015; 41(4):457-63.

9. Ellinger RF, Kelly WH. Maxillary sinus lymphoma: a consideration in the diagnosis of odontogenic pain. $\mathbf{J}$ Endod. 1989; 15(2):90-3.

10. Bavitz JB, Patterson DW, Sorensen S. Non-Hodgkin's lymphoma disguised as odontogenic pain. J Am Dent Assoc. 1992; 123(3):99-100.

11. Saund D, Kotecha S, Rout J, Dietrich T. Non-resolving periapical inflammation: a malignant deception. Int Endod J. 2010; 43(1):84-90.

12. Favia G, Maiorano E, Orsini G, Piattelli A. Central (intraosseous) adenoid cystic carcinoma of the mandible: report of a case with periapical involvement. J Endod. 2000; 26(12):760-3.

13. August M, Faquin W, Troulis M, Kaban L. Clear cell odontogenic carcinoma: evaluation of reported cases. J Oral Maxillofac Surg. 2003;61(5):580-6.
14. Kwon IJ, Kim SM, Amponsah EK, Myoung H, Lee JH, Lee SK. Mandibular clear cell odontogenic carcinoma. World J Surg Oncol. 2015;13:284.

15. Ino $\mathrm{M}$, Ishikawa $\mathrm{S}$, Ozaki $\mathrm{H}$, Kobayashi $\mathrm{T}$, Tachibana $\mathrm{H}$, Sakurai $\mathrm{H}$ et al. Solid type primary intraosseous squamous cell carcinoma in the maxilla: report of a new case. BMC Ear Nose Throat Disord. 2013; 13(1):13.

16. Sivolella S, Rizzo G, Valente M, Lumachi F. Sporadic burkitt lymphoma mimicking osteomyelitis of the mandible revealing clinically unsuspected HIV infection. Anticancer Res. 2015; 35(9):4837-9.

17. Prakash S, Shah ND, Chavda BV. Unnecessary extractions in patients with hemicrania continua: case reports and implication for dentistry. J Orofac Pain. 2010; 24(4):408-11.

18. Mello LA, Gurgel CA, Ramos EA, de Souza RO, Schlaepfer-Sales CB, de Azevedo RA, et al. Keratocystic odontogenic tumour: an experience in the Northeast of Brazi. Srp Arh Celok Lek. 2011; 139(5-6):291-7.

19. Sethukumar P, Taghi A, Kuchai R. A rare case of bilateral nasolabial cysts. BMJ Case Rep. 2015;203543.

20. Gelpi F, Romoli L, D'Arienzo D, Mazzoleni S, Santini S, Rossetto A et al. Lateral periodontal cyst (LPC) mimicking periodontal socket in a young patient: report of a case and review of the literature. Minerva Stomatol. 2014 Jan 14. [Epub ahead of print]

21. Vasudevan S, Palle AR, Sylvia DS, Renuka V, Challa R. A rare case of temporal arteritis with rheumatoid arthritis and interstitial lung disease mimicking pulpoperiodontal pathology. J Indian Soc Periodontol. 2014; 18(4):531-5.

22. Ahmadieh A, Farnad F, Sedghizadeh PP. Gaucher disease with jawbone involvement: a case report. J Med Case Rep. 2014; 8:360.

23. Mohammed I, Jannan NA, Elrmali A. Osteoid osteoma associated with the teeth: unusual presentation. Int $\mathbf{J}$ Oral Maxillofac Surg. 2013; 42(2):298-302.

24. Ribera MJ. Osteoblastoma in the anterior maxilla mimicking periapical pathosis of odontogenic origin. $\mathbf{J}$ Endod. 1996; 22(3):142-6.

25. López-López J, Garcia-Vicente L, Jané-Salas E, Estrugo-Devesa A, Chimenos-Küstner E, Roca-Elias J. Orofacial pain of cardiac origin: review literature and clinical cases. Med Oral Patol Oral Cir Bucal. 2012;17:538-44.

26. Sulfaro MA, Gobetti JP. Occipital neuralgia manifesting as orofacial pain. Oral Surg Oral Med Oral Pathol Oral Radiol Endod. 1995; 80(6):751-5.

27. Law AS, Lilly JP. Trigeminal neuralgia mimicking odontogenic pain. A report of two cases. Oral Surg Oral Med Oral Pathol Oral Radiol Endod. 1995; 80(1):96100.

28. Heir GM. Comparison of two patients with similar facial pain complaints of dental and non-dental etiologies. Cranio. 1995; 13(2):128-30. 


\section{CONFLITO DE INTERESSES}

Os autores declaram não haver conflitos de interesse.

\section{AUTOR PARA CORRESPONDÊNCIA}

José Alcides Almeida de Arruda alcides_almeida@hotmail.com

Submetido em 25/10/2017

Aceito em 17/11/2017 